\title{
Chiral phonons and pseudoangular momentum in nonsymmorphic systems
}

\author{
Tiantian Zhang (ㄷ) and Shuichi Murakami $\odot$ \\ Department of Physics, Tokyo Institute of Technology, Ookayama, Meguro-ku, Tokyo 152-8551, Japan \\ and Tokodai Institute for Element Strategy, Tokyo Institute of Technology, Nagatsuta, Midori-ku, Yokohama, Kanagawa 226-8503, Japan
}

(Received 14 July 2021; revised 9 January 2022; accepted 2 February 2022; published 22 February 2022)

\begin{abstract}
Chiral phonons have nonzero polarization and can be observed only via a selective coupling with valley electrons and circularly polarized photons. In such process, a new physical quantity, i.e., pseudoangular momentum (PAM), is required to meet the selection rule. However, phonon PAM was only studied in symmorphic systems with quantized integer values. In this work, we generalized the definition of PAM to systems with screw rotation symmetry, i.e., nonsymmorphic systems, which show distinct properties compared with the one in symmorphic systems: (i) PAM can be nonintegers and $\mathbf{q}$ dependent but still be an observable quantity by experiments; (ii) phonon PAM and phonon angular momentum are not equal due the special Wyckoff position in the real space, in contrast to the previous works where most of the atoms are at rotation-invariant Wyckoff positions. Such new definition and discoveries can help us to obtain chiral phonons in a broader class of systems with a nonzero group velocity and to convey information like chirality and angular momentum in solids as expected. Materials are also offered to understand the new definition and for further experimental detection.
\end{abstract}

DOI: 10.1103/PhysRevResearch.4.L012024

Introduction. Chirality has been widely studied in fundamental physics since it reveals symmetry breaking of (quasi-)particles and governs many unconventional phenomena, such as chiral anomaly [1-3], unconventional Landau spectrum [4,5], Klein tunneling [6], nontrivial surface states [7-17], and so on. In recent years, chiral phonons draw much attention due to their contribution to many physical processes, such as electronic phase transition $[18,19]$, giant thermal Hall effects in cuprate high- $T_{c}$ superconductors [20,21], and valley polarization [22-29]. Chiral phonons can also transport information like chirality and angular momentum if they further couple with valley electrons and photons. However, most of them were only studied in two-dimensional (2D) systems at either the Brillouin zone (BZ) center or its boundaries [30-37], which means that they will have a vanishing group velocity due to the local extremal energy and reduces the efficiency of the information propagation. Thus, studies on chiral phonons in three-dimensional (3D) systems with a nonvanishing group velocity will help us to realize the chiral phonon-based quantum devices through materials.

Pseudoangular momentum (PAM) is proposed as a quantized physical quantity defined by the eigenvalue of a rotation operator [38] to offer a crucial selection rule to study chiral phonon absorption/emission in the intra-/intervalley electron scattering process via photon absorption. Although chiral phonons at high-symmetry points (HSPs) were observed by the helicity-resolved Raman scattering experiments in

Published by the American Physical Society under the terms of the Creative Commons Attribution 4.0 International license. Further distribution of this work must maintain attribution to the author(s) and the published article's title, journal citation, and DOI. transition-metal dichalcogenides and graphene by detecting the helicity flipping of the incident and emission photons [34,35,39-44], such a definition restricts the study of chiral phonons only in symmorphic space groups [45] and PAM was only studied in systems with rotation symmetry. In this work, we propose that PAM can be nonintegers and $\mathbf{q}$ dependent at any momenta with (screw) rotation symmetry, even in the nonsymmorphic systems (except for No. 7 and No. 9, which only have a glide mirror symmetry), which will be much easier to obtain chiral phonons with nonzero group velocity to convey information along expected directions. In most cases studied in previous works, all the atoms are $C_{n}$-rotation invariant Wyckoff positions (WPs), which we call case I, and the possible patterns of atomic motions are limited and simple. An atom at a $C_{n}$-rotation invariant position means that the position of the atom will be invariant under the $C_{n}$ rotation operator. Other cases are classified into case II, and screw-symmetric systems always belong to this case II, having richer varieties than case I.

Chiral phonons and pseudoangular momentum. The chirality of phonons can be understood from the phonon polarization point of view, and regarded as one of the pieces of information carried by phonons. In 2D materials, phonon circular polarization for each mode is along the out-of-plane direction, such as $\mathrm{WSe}_{2}$ in Figs. 1(a) and 1(b), while in 3D materials it can point to any direction due to the interatomic coupling in all directions. Yet, we can still define the phonon polarization, i.e., phonon chirality, for a 3D system by the vibration trajectory of atoms in the real space: if the atom vibrates in a counterclockwise circular/elliptical trajectory on a 2D plane, it will carry a counterclockwise chirality labeled by "+," as shown in Fig. 1(e); otherwise, it will carry a clockwise chirality labeled by "-,, as shown in Fig. 1(f). 
(a)top view of $\mathrm{WSe}_{2}, \mathrm{I}^{\circ}=-1$ for $\mathrm{W}$ atom (b) top view of $\mathrm{WSe}_{2}, \mathrm{I}^{\circ}=+1$ for Se atom
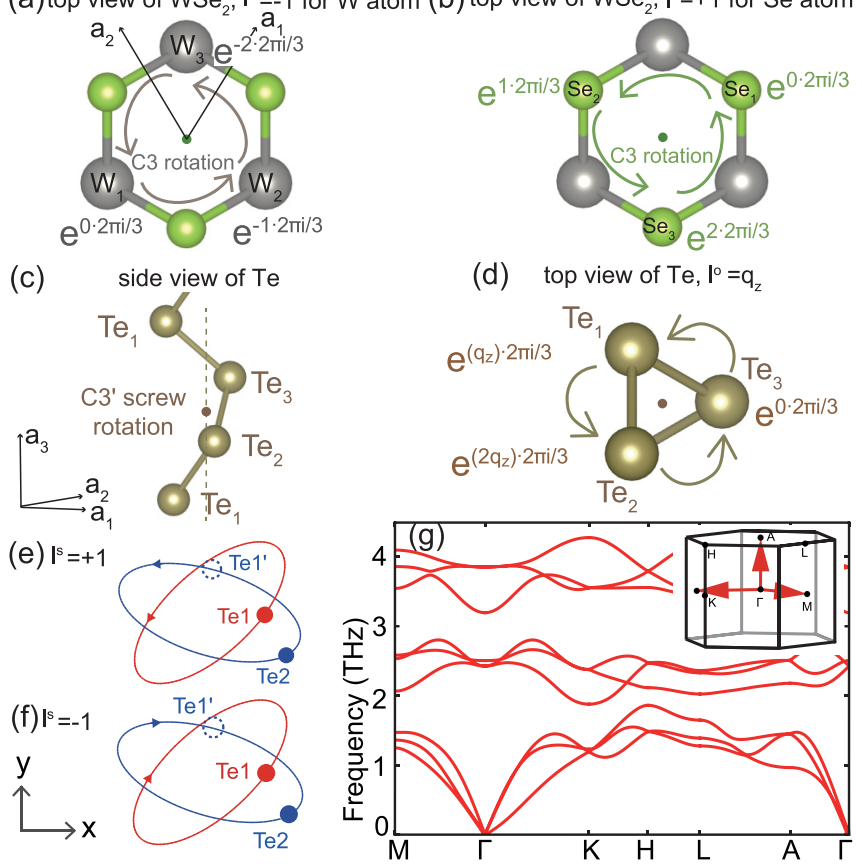

FIG. 1. (a), (b) Phase change of the phonon nonlocal part at $K$ $\left(\frac{1}{3}, \frac{1}{3}\right)$ under $\hat{C}_{3}$ for $2 \mathrm{D}$ material $\mathrm{WSe}_{2} . l^{o}=-1$ for the $\mathrm{W}$ atom in (a), while $l^{\circ}=+1$ for the Se atom in (b). (c), (d) Side and top views of the 3D material Te with screw rotation symmetry $\hat{C}_{3}^{\prime}$. (d) Phase change of the phonon nonlocal part for Te at $\left(\frac{1}{3}, \frac{1}{3}, q_{z}\right)$ under $\hat{C}_{3}^{\prime}$, which corresponds to $l^{o}=+q_{z}$. (e), (f) Schematic figures for two different vibration trajectories of the third and first modes for Te along the $K$ - $H$ high-symmetry line $\left(\frac{1}{3}, \frac{1}{3}, \frac{1}{4}\right)$, which have an intracell part of pseudangular momentum with $l^{s}=+1,-1$, respectively. (g) Phonon spectra and $\mathrm{BZ}$ of Te.

As a selection rule for the optical transition process, PAM offers an additional conservation condition besides the crystal momentum conservation and energy conservation in the valley-dependent optoelectronics:

$$
\begin{gathered}
l_{e}^{v}+m=l_{e}^{c}+l_{p h}, \\
\hbar \mathbf{k}_{e}^{v}=\hbar \mathbf{k}_{e}^{c}+\hbar \mathbf{q}_{p h}, \\
E_{e}^{v}+E_{\text {photon }}=E_{e}^{c}+E_{p h},
\end{gathered}
$$

where $l_{e}^{v / c}$ is the electron PAM for the valence/conduction band, $l_{p h}$ is the PAM for a phonon, $m= \pm 1$ represent the right/left-circularly polarized light, $\mathbf{k}_{e}$ and $\mathbf{q}_{p h}$ are the crystal momentum for electrons and phonons, and $E_{e / \text { photon/ph }}$ is the energy for electrons/photons/phonons.

The PAM was only studied in symmorphic space groups, in terms of the eigenvalue of $n$-fold rotation symmetry:

$$
\hat{C}_{n} u_{\mathbf{q}}=e^{(-2 \pi i / n) l_{p h, \mathbf{q}}} u_{\mathbf{q}},
$$

where $\hat{C}_{n}$ is the $n$-fold rotation operator, $u_{\mathbf{q}}$ is the phonon Bloch wave function, and $l_{p h, \mathbf{q}}$ is the phonon PAM at $\mathbf{q}$ with discrete values of $\{0,1, \ldots, n-1\} \bmod n$. We propose that PAM is still well defined and $\mathbf{q}$ dependent ( $\mathbf{k}$ dependent for electrons) for nonsymmorphic systems with screw rotation symmetry via Eq. (4) with $\hat{C}_{n}$ replaced by the screw rotation operator $\hat{C}_{n}^{\prime}$. The definition can be extended for any (quasi-) particles at any momenta in solids preserving (screw) rotation symmetry.

Definition for intercell and intracell parts of PAM. Since the phonon Bloch wave function $u_{\mathbf{q}}$ has both a nonlocal contribution (Bloch-phase part $l^{o}$, which is also called intercell/orbital part in the previous works) from the Bloch phase factor $e^{i \cdot \mathbf{R}_{l} \cdot \mathbf{q}}$ $\left(\mathbf{R}_{l}\right.$ is the atomic position) and a local contribution (selfrotation part $l^{s}$, which is also called intracell/spin part) from relative vibrations between sublattices, phonon PAM can be decomposed into $l_{p h}=l^{s}+l^{o}$ [46]. Like the total PAM, $l^{o}$ and $l^{s}$ should be also defined along the (screw) rotation axis direction. We find that motions of atoms for each phonon mode are totally different between two classes of systems, such as whether all the atoms are (screw) rotation-preserved sites (case I) or not (case II). In case I, all of the atoms are at the rotation-invariant WPs, and the value of $l^{\circ}$ is uniquely determined by the WPs and the wave vector $\mathbf{q}$ (see the Supplemental Material for details [47]). We show that the possible combination of $l^{o}$ and $l^{s}$ is limited, because $l_{p h}=l^{s}+l^{o}$ is common for all the atoms. PAM equals AM when $\mathbf{q}=0$ in case I. But in case II and some $\mathbf{q} \neq 0$ cases in case I, PAM does not equal AM. Cases in the previous works which belong to case I are among the list in the Supplemental Material [47], but such discussions in case II have not been done before. Thus, we will focus on case II in the following, and use Te as an example to show how to obtain $l^{o}$ and $l^{s}$. It is worth mentioning that case II is the only possibility for nonsymmorphic systems with screw rotation symmetry.

The intercell/orbital part of PAM, i.e., $l^{o}$, is the phase difference of the Bloch phase factor between atoms in different sublattices related by (screw) rotation symmetry, and it depends on the phonon momentum $\mathbf{q}$ and the WP $\mathbf{R}_{l}$ in the real space. $l^{o}$ is a quantized integer for symmorphic systems, while it becomes a q-dependent quantity for systems with screw rotation symmetry due to the fractional translation. For example, Figs. 1(a) and 1(b) show the phases of the phonon nonlocal part $e^{i \cdot \mathbf{R}_{l} \cdot \mathbf{q}}$ at $\mathbf{q}=K\left(\frac{1}{3}, \frac{1}{3}\right)$ for $\mathrm{W}$ and $\mathrm{Se}$ in $\mathrm{WSe}_{2}$, which belongs to case I. We note that all the coordinates in the real and reciprocal space are expressed in the basis of the primitive lattice vectors shown in Figs. 1(a) and 1(c). We take the $\hat{C}_{3}$ center to be at the center of the hexagon, which is the WP $1 a$. The W atom and Se atom are at WPs $1 b$ and $1 c$, respectively (see the Supplemental Material for details [47]). The tungsten atom has $l_{W}^{o}=-1$ from the phase difference among the three $\hat{C}_{3}$-related atoms in Fig. 1(a). Likewise, the selenium atom has $l_{\mathrm{Se}}^{o}=+1$ in Fig. 1(b). This leads to $l_{p h}=l_{\mathrm{W}}^{o}+l_{\mathrm{W}}^{s}=l_{\mathrm{Se}}^{o}+l_{\mathrm{Se}}^{s}$, i.e., $l_{\mathrm{W}}^{s}=l_{\mathrm{Se}}^{s}+2 \bmod 3$, meaning that $\left(l_{\mathrm{W}}^{s}, l_{\mathrm{Se}}^{s}\right)=(1,-1),(0,1),(-1,0)$, which agree with the combinations in the ab initio calculations in Ref. [34]. On the other hand, case II is the only possibility in systems with screw rotation symmetry. For example, Figs. 1(c), 1(d) and $1(\mathrm{~g})$ are the crystal and phonon band structure of Te, which has a nonsymmorphic crystal structure $P 3_{1} 21$ (No. 152) with a threefold screw rotation $\left\{C_{3 z}^{\prime}=C_{3 z} \mid\left(0,0, \frac{1}{3}\right)\right\}$. Figure 1(d) shows the phases of the phonon nonlocal part $e^{i \cdot \mathbf{R}_{l} \cdot \mathbf{q}}$ at $\mathbf{q}=$ $\left(\frac{1}{3}, \frac{1}{3}, q_{z}\right)$, and the fractional atomic positions for the three ${\hat{C_{3 z}^{\prime}}}^{-}$ related tellurium atoms are $\mathbf{R}_{\mathbf{1}}=(x, x, 0), \mathbf{R}_{\mathbf{2}}=\left(-x, 0, \frac{1}{3}\right)$, and $\mathbf{R}_{\mathbf{3}}=\left(0,-x, \frac{2}{3}\right)$. Thus, $\left(e^{-i(2 \pi / 3) l^{\circ}}\right)^{3}=e^{-i \cdot \mathbf{q} \cdot \mathbf{R}_{0}}=e^{-2 \pi i \cdot q_{z}}$, 
i.e., $l^{o}=q_{z}$ for all the phonon modes in Te according to the phase difference, where $\mathbf{R}_{\mathbf{0}}$ is the unit lattice vector along the $c$ axis.

The intracell/spin part of PAM, $l^{s}$, which is a local contribution from the sublattice relative vibration, depends on the in-plane atomic vibration. In case I, $l^{s}=1$ and $l^{s}=-1$ correspond to counterclockwise and clockwise rotations within the $x y$ plane, and when $l^{o}=0, l^{s}$ can directly correspond to the AM. Since the possible situations for case I are very limited due to the symmetry restriction in both real and reciprocal space, we list all the possibilities in the Supplemental Material [47]. However, it will become much more complicated in case II, where $l^{s}$ represents relative motions between the $C_{n}$-related atoms, instead of the atomic motion. In other words, $l^{s}$ is the real space motion of a cluster, which is composed of all the $C_{n}$ related atoms. Thus, we need to calculate the phase difference between atoms related by screw rotation symmetry in nonsymmorphic systems. For example, Figs. 1(e) and 1(f) show the vibration trajectories of the third and first phonon bands for two $\hat{C}_{3}^{\prime}$-related tellurium atoms at the middle point of the $K-H$ line $\left(\frac{1}{3}, \frac{1}{3}, \frac{1}{4}\right)$, respectively, where both of those tellurium atoms have a counterclockwise polarization. In Fig. 1(e), $\mathrm{Te}_{1}^{\prime}$, which is obtained from $\mathrm{Te}_{1}$ through the screw rotation $\hat{C}_{3}^{\prime}$, has a $\frac{2 \pi}{3}$ phase advancing to $\mathrm{Te}_{2}$ and therefore this case corresponds to $l^{s}=+1$ for Te.

Distinct features of PAM in case II with screw rotation symmetry. We note that the definition of PAM in nonsymmorphic systems is not just an extension of that in symmorphic systems, but has unique features: (i) The noninteger nature of $l_{p h}$ is from the fractional-translation part of the screw rotation symmetry, which is unique for nonsymmorphic systems. Namely, the phase difference between sublattices under screw rotation symmetry leads to a noninteger value of $l^{o}$. (ii) We only need to consider the atomic position components along the screw rotation axis when calculating $l^{o}$, which means that the atomic positions in plane will make no influence on $l^{\circ}$. (iii) Case II which we propose is the only possibility for nonsymmorphic systems with screw rotation symmetry. In the following, we will use nonsymmorphic crystal Te as an example to show those distinct features.

Electronic band structure of Te. Figure 3(a) is the spinful electronic band structure of $\mathrm{Te}\left(P 3_{1} 21\right)$ with experimental lattice constants [48], and it shows that $\mathrm{Te}$ is a narrow gap insulator with a direct gap about $\Delta=0.3 \mathrm{eV}$ at $P\left(\frac{1}{3}, \frac{1}{3}, q\right)$ and $P A\left(-\frac{1}{3},-\frac{1}{3},-q\right)$ in the vicinity of $H$ [49]. Thus, chiral phonons involved in the valley scattering will be also away from HSPs and take values of $l_{p h, \mathbf{q}}=1+q_{z}, q_{z}, q_{z}-1 \mathrm{mod}$ 3 with $q_{z} \in[-0.5,0.5]$. The highest two valence bands have a very large spin splitting about $0.18 \mathrm{eV}$, while the lowest two conduction bands have a spin splitting about $30 \mathrm{meV}$. Moreover, the spin component is different for the valance band maximum (VBM) and the conduction band minimum (CBM) at two valleys $P$ and $P A$, which forbids the intravalley transition of electrons at each valley. Thus, both the multivalley band structure and different spin components for the VBM and CBM bands will be advantageous to realize selective coupling between chiral phonons and valley electrons via photon absorption. (a) phonon bands in the first $B Z:\left.\right|^{s}=0,+1,-1 ; l^{0}=q_{z} ; l_{p h}=q_{z}, q_{z}+1, q_{z}-1$

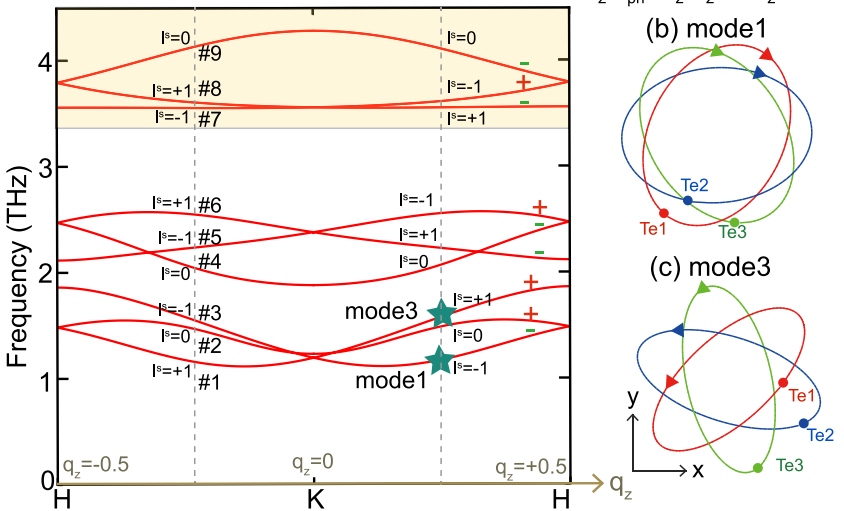

(d) top three phonon bands in the extended BZ: $I^{\mathrm{s}}=0$; $\mathrm{I}^{\circ}=\mathrm{w} ; \mathrm{I}_{\mathrm{ph}}=\mathrm{w}$

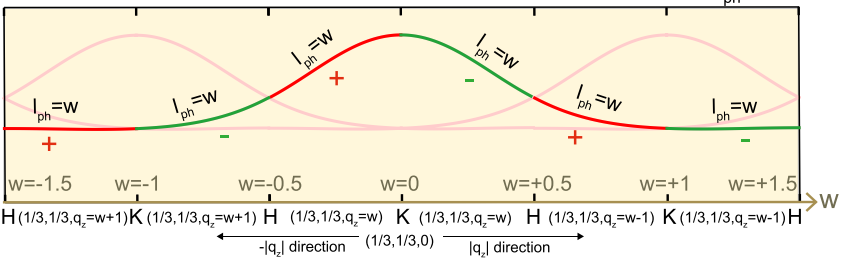

FIG. 2. (a) Phonon spectra of Te along the $H-K-H\left(\frac{1}{3}, \frac{1}{3}, q_{z}\right)$ direction in the first BZ, where $q_{z} \in[-0.5,0.5]$. "+" and "-" represent the chirality and $l^{s}$ is the intracell part of the phonon angular momentum for each mode. Both the phonon chirality and $l^{s}$ will keep the same value for each continuous phonon band. Real space trajectories for (b) the first and (c) the third mode at $\left(\frac{1}{3}, \frac{1}{3}, \frac{1}{4}\right)$ with $l^{s}=-1$ and $l^{s}=+1$, respectively. (d) The highest three phonon modes along the $H-K-H\left(\frac{1}{3}, \frac{1}{3}, w\right)$ direction crossing three extended $\mathrm{BZs}$, where $l^{s}=0, l^{o}=l_{p h}=w$, and $w \in[-1.5,1.5]$.

PAM of Te. Figure 2(a) shows the chirality and $l^{s}$ for each phonon band in the first BZ. $l^{s}$ takes the values $0, \pm 1$ and keeps the same value for each continuous band, while $l^{o}=q_{z} \in[-0.5,0.5]$ is a $\mathbf{q}$-dependent quantity. Thus, $l_{p h}=$ $l^{s}+l^{o}$, taking the values $q_{z}, q_{z} \pm 1$, is also a q-dependent quantity. Consequently, $l^{s}$ changes by +1 when $q_{z}$ increases across the BZ boundary at $H$ due to the $\hat{C}_{3}^{\prime}$ screw symmetry. Table I shows the results of $l^{o}, l^{s}$, and $l_{p h}$ for all the phonon modes at $\left(\frac{1}{3}, \frac{1}{3}, \frac{1}{4}\right)$, and $l_{p h}$ can be calculated by two ways: (i) the summation of the intracell and intercell parts of PAM, i.e., $l_{p h}=l^{o}+l^{s}$; (ii) $l_{p h}$ is calculated directly from the eigenvalue of $\hat{C_{3 z}^{\prime}}$.

Since nine phonon modes are well separated into three groups in Fig. 2(a), each group can be understood as a result of band folding from a single phonon band extending over three BZs, and the values of $l^{s}, l^{o}$, and $l_{p h}$ can be

TABLE I. $l^{o}, l^{s}$, and $l_{p h}$ for each phonon band at $\left(\frac{1}{3}, \frac{1}{3}, \frac{1}{4}\right)$ in tellurium, which is the middle point of the $K-H$ line.

\begin{tabular}{lcccc}
\hline \hline Band order & \multicolumn{1}{c}{$l^{s}$} & $l^{o}$ & $\begin{array}{c}l_{p h} \text { calculated } \\
\text { by } l^{s}+l^{o}\end{array}$ & $\begin{array}{c}l_{p h} \text { calculated by } \\
\text { eigenvalue of } \hat{C}_{3}^{\prime}\end{array}$ \\
\hline No. 1, No. 6, No. 8 & -1 & 0.25 & -0.75 & -0.75 \\
No. 2, No. 4, No. 9 & 0 & 0.25 & 0.25 & 0.25 \\
No. 3, No. 5, No. 7 & +1 & 0.25 & 1.25 & 1.25 \\
\hline \hline
\end{tabular}


understood in an extended scheme over three BZs [50]. Figure 2(d) shows the top three phonon bands [No. 7, No. 8, and No. 9 in Fig. 2(a)] in the extended three BZs, where the horizontal axis is the momentum along the $H-K$ direction with $\left(\frac{1}{3}, \frac{1}{3}, w\right)$ and $w \in[-1.5,1.5]$. There is only one optical phonon band from the vibration of one tellurium in the extended $\mathrm{BZ}$, which leads to a trivial value of $l^{s}=0, l^{o}=w$, and $l_{p h}=l^{s}+l^{o}=w$. By noting that the wave number $w \in$ $[-1.5,1.5]$ along the $z$ axis in the extended scheme is related to that in the reduced scheme, $q_{z} \in[-0.5,0.5]$, via $q_{z}=w$ (mod 1), the result in Fig. 2(a) immediately follows from Fig. 2(d). Figures 2(b) amd 2(c) show the trajectories of two $C_{3 z}^{\prime}$-related atoms for the first and third phonon modes, which is consistent with the $l^{s}$ calculation explained in Figs. 1(e) and $1(\mathrm{f})$.

We note that Te has another chiral crystal structure of $P 3_{2} 21$ (No. 154) with $\left\{C_{3 z}^{\prime}=C_{3 z} \mid\left(0,0,-\frac{1}{3}\right)\right\}$, which will have the same phonon band spectra as $P 3_{1} 21$. However, $l_{p h}, l^{o}$, and $l^{s}$ will have an additional sign compared to the ones in $P 3_{1} 21$ due to the opposite fractional translation along the $z$ direction. Furthermore, $l_{p h}, l^{o}$, and $l^{s}$ can be defined in the same way if systems have higher symmetries and higher degenerated modes. In systems with $n_{m}$ screw symmetry, $l^{o}$ takes the value of $l^{o}=m q_{z} \bmod n$. Thus, when $q_{z}$ is increased across the BZ at $q_{z}= \pm \frac{1}{2}, l^{s}$ jumps by $m$.

Chiral phonon-involved valley scattering in Te. As discussed above, the spin conservation condition forbids the intravalley transition in Te but allows the intervalley one between the CBM and VBM. In such process, phonons will be involved in the conservation of the total crystal momentum, PAM, and energy:

$$
\begin{gathered}
\mathbf{q}_{p h}=\mathbf{k}_{e, P}-\mathbf{k}_{e, P A}, \\
l_{e, P}^{v}+m+l_{p h}=l_{e, P A}^{v} \bmod 3, \\
E_{\text {photon }}=E_{p h}+\Delta,
\end{gathered}
$$

where $m= \pm 1$ represent the right/left-circularly polarized light and $\Delta$ is the electronic band gap of Te. We note that $m$ is also not a quantized value due to the fractional translation of the screw rotation. However, we take $m= \pm 1$ since the fractional translation of the crystal is negligible compared to the wavelength of the photon.

Figures 3(b) and 3(c) showthe chiral phonon absorption and emission process, and we will take the former one as an example. Since the momentum difference for electrons in the intervalley transition is $\mathbf{q}_{p h}=\mathbf{k}_{e, P}-\mathbf{k}_{e, P A}=\left(\frac{2}{3}, \frac{2}{3}, 2 q\right)$, phonons with momentum of $\mathbf{q}_{p h}=\left(\frac{2}{3}, \frac{2}{3}, 2 q\right)$ will be involved in this process with possible PAM values of $l_{p h}=2 q$, $2 q+1$, and $2 q-1$. Furthermore, $l_{e}$ can be obtained from the eigenvalue of ${\hat{C_{3 z}^{\prime}}}_{3}$ at valley $P$ and $P A$ by first-principles calculation, i.e., $l_{e, P}^{v}=q-\frac{1}{2}, l_{e, P A}^{v}=-q+\frac{1}{2}, l_{e, P}^{c}=q-\frac{3}{2}$, $l_{e, P A}^{c}=-q+\frac{3}{2}$. Thus, by following Eq. (6), a chiral phonon

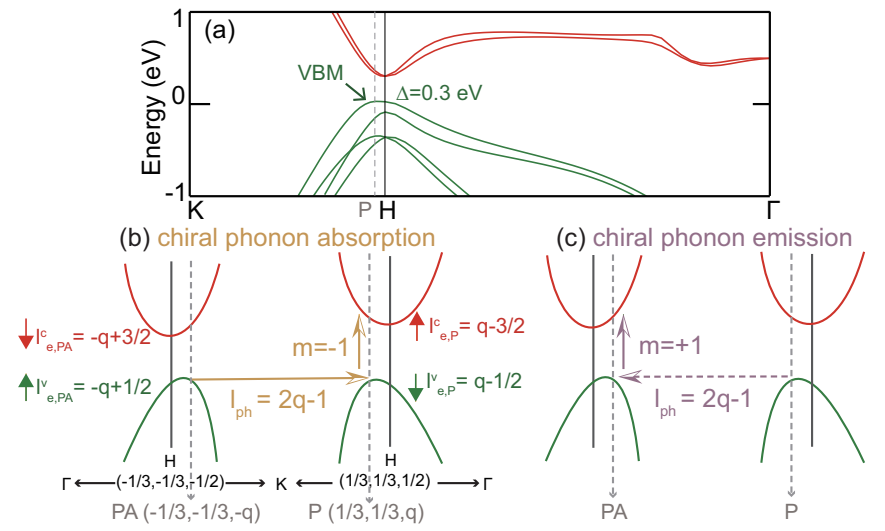

FIG. 3. New selection rules for phonon-involved intervalley scattering in 3D nonsymmorphic crystal Te. (a) Spinful electronic band structure of Te with a direct gap at $P\left(\frac{1}{3}, \frac{1}{3}, q\right)$. (b) By absorbing a chiral phonon with $l_{p h}=2 q-1$, electrons can be scattered between $P$ and $P A$ valleys under the left-circularly polarized light $(m=-1)$. (c) By emitting a chiral phonon with $l_{p h}=2 q-1$, electrons can be scattered between two valleys under the right-circularly polarized light $(m=+1)$.

absorption process can be obtained with $l_{p h}=2 q-1$ by a left-circularly polarized incident photon with an energy $\Delta$, as shown in Fig. 3(b). (Electrons can be scattered between those two valleys by emitting a chiral phonon with $l_{p h}=2 q-1$ and $m=+1$, and they can also be scattered between other valleys by absorbing/emitting chiral phonons with different PAM.)

Conclusion. In this work, we not only generalize the definition of PAM (including $l^{s}$ and $l^{o}$ ) to nonsymmorphic space groups at arbitrary momenta with screw rotation symmetry, but also discuss the more unique features of PAM that the screw rotation-preserved systems have than the symmorphic ones, which is due to the special WPs in the real space. The redefinition not only makes us realize that PAM can be nonintegers and $\mathbf{q}$ dependent, but also helps us to obtain chiral phonons with a broader condition. For example, $\mathrm{Na}_{3} \mathrm{Sb}$ is a direct gap semiconductor with large Fermi velocity for the valence band maximum along different directions in the vicinity of $A$, which has the same crystal structure with Dirac semimetal $\mathrm{Na}_{3} \mathrm{Bi}$ [51]. Since $\mathrm{Na}_{3} \mathrm{Sb}$ preserves $\hat{C}_{6}^{\prime}$ symmetry $\left(P 6_{3} / m m c\right)$, noninteger chiral phonons with PAM $\in[-3,3]$ mod 6 can be observed by experiments if one gradually modulates the energy of the incident light to excite the electrons along the $A-\Gamma$ direction [47].

Acknowledgments. We acknowledge L.F. Zhang and $\mathrm{H}$. Chen for discussion. We acknowledge the supports from Tokodai Institute for Element Strategy (TIES) funded by MEXT Elements Strategy Initiative to Form Core Research Center Grant No. JPMXP0112101001, Japan Society for the Promotion of Science (JSPS) KAKENHI Grants No. JP18H03678 and No. JP20H04633. T.Z. also acknowledge the support by JSPS KAKENHI Grant No. JP21K13865.
[1] H. B. Nielsen and M. Ninomiya, The Adler-Bell-Jackiw anomaly and Weyl fermions in a crystal, Phys. Lett. B 130, 389 (1983).
[2] D. T. Son and B. Z. Spivak, Chiral anomaly and classical negative magnetoresistance of Weyl metals, Phys. Rev. B 88, 104412 (2013). 
[3] X. Huang, L. Zhao, Y. Long, P. Wang, D. Chen, Z. Yang, H. Liang, M. Xue, H. Weng, Z. Fang et al., Observation of the Chiral-Anomaly-Induced Negative Magnetoresistance in 3D Weyl Semimetal TaAs, Phys. Rev. X 5, 031023 (2015).

[4] Y. Zhang, Y.-W. Tan, H. L. Stormer, and P. Kim, Experimental observation of the quantum Hall effect and Berry's phase in graphene, Nature (London) 438, 201 (2005).

[5] Y. X. Zhao and S. A. Yang, Index Theorem on Chiral Landau Bands for Topological Fermions, Phys. Rev. Lett. 126, 046401 (2021).

[6] M. I. Katsnelson, K. S. Novoselov, and A. K. Geim, Chiral tunnelling and the Klein paradox in graphene, Nat. Phys. 2, 620 (2006).

[7] T. Zhang, Z. Song, A. Alexandradinata, H. Weng, C. Fang, L. $\mathrm{Lu}$, and Z. Fang, Double-Weyl Phonons in Transition-Metal Monosilicides, Phys. Rev. Lett. 120, 016401 (2018).

[8] L. Lu, J. D. Joannopoulos, and M. Soljačić, Topological photonics, Nat. Photonics 8, 821 (2014).

[9] L. Lu, Z. Wang, D. Ye, L. Ran, L. Fu, J. D. Joannopoulos, and M. Soljačić, Experimental observation of Weyl points, Science 349, 622 (2015).

[10] H. Weng, C. Fang, Z. Fang, B. Andrei Bernevig, and X. Dai, Weyl Semimetal Phase in Noncentrosymmetric TransitionMetal Monophosphides, Phys. Rev. X 5, 011029 (2015).

[11] B. Bradlyn, J. Cano, Z. Wang, M. G. Vergniory, C. Felser, R. J. Cava, and B. Andrei Bernevig, Beyond Dirac and Weyl fermions: Unconventional quasiparticles in conventional crystals, Science 353, aaf5037 (2016).

[12] B. Q. Lv, H. M. Weng, B. B. Fu, X. P. Wang, H. Miao, J. Ma, P. Richard, X. C. Huang, L. X. Zhao, G. F. Chen et al., Experimental Discovery of Weyl Semimetal TaAs, Phys. Rev. X 5, 031013 (2015).

[13] S.-Y. Xu, I. Belopolski, N. Alidoust, M. Neupane, G. Bian, C. Zhang, R. Sankar, G. Chang, Z. Yuan, C.-C. Lee et al., Discovery of a Weyl fermion semimetal and topological Fermi arcs, Science 349, 613 (2015).

[14] X. Wan, A. M. Turner, A. Vishwanath, and S. Y. Savrasov, Topological semimetal and Fermi-arc surface states in the electronic structure of pyrochlore iridates, Phys. Rev. B 83, 205101 (2011).

[15] D. Zeb Rocklin, Bryan Gin-ge Chen, M. Falk, V. Vitelli, and T. C. Lubensky, Mechanical Weyl Modes in Topological Maxwell Lattices, Phys. Rev. Lett. 116, 135503 (2016).

[16] M. Xiao, W.-J. Chen, W.-Y. He, and C. T. Chan, Synthetic gauge flux and Weyl points in acoustic systems, Nat. Phys. 11, 920 (2015).

[17] C. He, X. Ni, H. Ge, X.-C. Sun, Y.-B. Chen, M.-H. Lu, X.-P. Liu, and Y.-F. Chen, Acoustic topological insulator and robust one-way sound transport, Nat. Phys. 12, 1124 (2016).

[18] M. Rini, N. Dean, J. Itatani, Y. Tomioka, Y. Tokura, R. W. Schoenlein, A. Cavalleri et al., Control of the electronic phase of a manganite by mode-selective vibrational excitation, Nature (London) 449, 72 (2007).

[19] M. Forst, R. Mankowsky, and A. Cavalleri, Mode-selective control of the crystal lattice, Acc. Chem. Res. 48, 380 (2015).

[20] G. Grissonnanche, S. Thériault, A. Gourgout, M.-E. Boulanger, E. Lefrançois, A. Ataei, F. Laliberté, M. Dion, J.-S. Zhou, S. Pyon et al., Chiral phonons in the pseudogap phase of cuprates, Nat. Phys. 16, 1108 (2020).
[21] G. Grissonnanche, A. Legros, S. Badoux, E. Lefrançois, V. Zatko, M. Lizaire, F. Laliberté, A. Gourgout, J.-S. Zhou, S. Pyon et al., Giant thermal Hall conductivity in the pseudogap phase of cuprate superconductors, Nature (London) 571, 376 (2019).

[22] H. Zeng, J. Dai, W. Yao, D. Xiao, and X. Cui, Valley polarization in $\mathrm{MoS}_{2}$ monolayers by optical pumping, Nat. Nanotechnol. 7, 490 (2012).

[23] B. R. Carvalho, Y. Wang, S. Mignuzzi, D. Roy, M. Terrones, C. Fantini, V. H. Crespi, L. M. Malard, and M. A. Pimenta, Intervalley scattering by acoustic phonons in two-dimensional $\mathrm{MoS}_{2}$ revealed by double-resonance Raman spectroscopy, Nat. Commun. 8, 14670 (2017).

[24] T. Cao, G. Wang, W. Han, H. Ye, C. Zhu, J. Shi, Q. Niu, P. Tan, E. Wang, B. Liu et al., Valley-selective circular dichroism of monolayer molybdenum disulphide, Nat. Commun. 3, 1 (2012).

[25] Q. H. Wang, K. Kalantar-Zadeh, A. Kis, J. N. Coleman, and M. S. Strano, Electronics and optoelectronics of two-dimensional transition metal dichalcogenides, Nat. Nanotechnol. 7, 699 (2012).

[26] K. He, N. Kumar, L. Zhao, Z. Wang, K. F. Mak, H. Zhao, and J. Shan, Tightly Bound Excitons in Monolayer WSe ${ }_{2}$, Phys. Rev. Lett. 113, 026803 (2014).

[27] S. Wu, J. S. Ross, G.-B. Liu, G. Aivazian, A. Jones, Z. Fei, W. Zhu, D. Xiao, W. Yao, D. Cobden et al., Electrical tuning of valley magnetic moment through symmetry control in bilayer $\mathrm{MoS}_{2}$, Nat. Phys. 9, 149 (2013).

[28] A. M. Jones, H. Yu, N. J. Ghimire, Sanfeng Wu, G. Aivazian, J. S. Ross, B. Zhao, J. Yan, D. G. Mandrus, D. Xiao et al., Optical generation of excitonic valley coherence in monolayer $\mathrm{WSe}_{2}$, Nat. Nanotechnol. 8, 634 (2013).

[29] X. Xu, W. Yao, D. Xiao, and T. F. Heinz, Spin and pseudospins in layered transition metal dichalcogenides, Nat. Phys. 10, 343 (2014).

[30] C. Chen, X. Chen, B. Deng, K. Watanabe, T. Taniguchi, S. Huang, and F. Xia, Probing interlayer interaction via chiral phonons in layered honeycomb materials, Phys. Rev. B 103, 035405 (2021).

[31] S. G. Drapcho, J. Kim, X. Hong, C. Jin, S. Shi, S. Tongay, J. $\mathrm{Wu}$, and F. Wang, Apparent breakdown of Raman selection rule at valley exciton resonances in monolayer $\mathrm{MoS}_{2}$, Phys. Rev. B 95, 165417 (2017)

[32] Y. Tatsumi and R. Saito, Interplay of valley selection and helicity exchange of light in Raman scattering for graphene and $\mathrm{MoS}_{2}$, Phys. Rev. B 97, 115407 (2018).

[33] L. M. Malard, M. A. Pimenta, G. Dresselhaus, and M. S. Dresselhaus, Raman spectroscopy in graphene, Phys. Rep. 473, 51 (2009).

[34] H. Zhu, J. Yi, M.-Y. Li, J. Xiao, L. Zhang, C.-W. Yang, R. A. Kaindl, L.-J. Li, Y. Wang, and X. Zhang, Observation of chiral phonons, Science 359, 579 (2018).

[35] X. Chen, X. Lu, S. Dubey, Q. Yao, S. Liu, X. Wang, Q. Xiong, L. Zhang, and A. Srivastava, Entanglement of single-photons and chiral phonons in atomically thin $\mathrm{WSe}_{2}$, Nat. Phys. 15, 221 (2019).

[36] Z. Li, T. Wang, C. Jin, Z. Lu, Z. Lian, Y. Meng, M. Blei, M. Gao, T. Taniguchi, K. Watanabe et al., Momentum-dark intervalley exciton in monolayer tungsten diselenide brightened via chiral phonon, ACS Nano 13, 14107 (2019). 
[37] Z. Li, T. Wang, C. Jin, Z. Lu, Z. Lian, Y. Meng, M. Blei, S. Gao, T. Taniguchi, K. Watanabe et al., Emerging photoluminescence from the dark-exciton phonon replica in monolayer $\mathrm{WSe}_{2}$, Nat. Commun. 10, 1 (2019).

[38] L. Zhang and Q. Niu, Chiral Phonons at High-Symmetry Points in Monolayer Hexagonal Lattices, Phys. Rev. Lett. 115, 115502 (2015).

[39] A. T. Hanbicki, H.-J. Chuang, M. R. Rosenberger, C. S. Hellberg, S. V. Sivaram, K. M. McCreary, I. I. Mazin, and B. T. Jonker, Double indirect interlayer exciton in a $\mathrm{MoSe}_{2} / \mathrm{WSe}_{2}$ van der Waals heterostructure, ACS Nano 12, 4719 (2018).

[40] J. Kunstmann, F. Mooshammer, P. Nagler, A. Chaves, F. Stein, N. Paradiso, G. Plechinger, C. Strunk, C. Schüller, G. Seifert et al., Momentum-space indirect interlayer excitons in transition-metal dichalcogenide van der Waals heterostructures, Nat. Phys. 14, 801 (2018).

[41] E. H. Martins Ferreira, M. V. O. Moutinho, F. Stavale, M. M. Lucchese, R. B. Capaz, C. A. Achete, and A. Jorio, Evolution of the Raman spectra from single-, few-, and many-layer graphene with increasing disorder, Phys. Rev. B 82, 125429 (2010).

[42] Y. Hao, Y. Wang, L. Wang, Z. Ni, Z. Wang, R. Wang, C. K. Koo, Z. Shen, and J. T. L. Thong, Probing layer number and stacking order of few-layer graphene by Raman spectroscopy, Small 6, 195 (2010).

[43] A. Eckmann, A. Felten, A. Mishchenko, L. Britnell, R. Krupke, K. S. Novoselov, and C. Casiraghi, Probing the nature of defects in graphene by Raman spectroscopy, Nano Lett. 12, 3925 (2012).

[44] Z. Ni, Y. Wang, T. Yu, and Z. Shen, Raman spectroscopy and imaging of graphene, Nano Res. 1, 273 (2008).

[45] H. Chen, W. Wu, J. Zhu, S. A. Yang, and L. Zhang, Propagating chiral phonons in three-dimensional materials, Nano Lett. 21, 3060 (2021).

[46] W. Yao, D. Xiao, and Q. Niu, Valley-dependent optoelectronics from inversion symmetry breaking, Phys. Rev. B 77, 235406 (2008).

[47] See Supplemental Material at http://link.aps.org/supplemental/ 10.1103/PhysRevResearch.4.L012024 for (i) all the possibilities of $l^{o}$ and $l^{s}$ in case I, (ii) electronic band structure of topological insulator $\mathrm{Na}_{3} \mathrm{Bi}$, and (iii) PAM in $\mathrm{Na}_{3} \mathrm{Bi}$.

[48] C. Adenis, V. Langer, and O. Lindqvist, Reinvestigation of the structure of tellurium, Acta Crystallogr. Sect. C: Cryst. Struc. Commun. 45, 941 (1989).

[49] M. Hirayama, R. Okugawa, S. Ishibashi, S. Murakami, and T. Miyake, Weyl Node and Spin Texture in Trigonal Tellurium and Selenium, Phys. Rev. Lett. 114, 206401 (2015).

[50] I. Božovic, Possible band-structure shapes of quasi-onedimensional solids, Phys. Rev. B 29, 6586 (1984).

[51] Z. Wang, Y. Sun, X.-Q. Chen, C. Franchini, G. Xu, H. Weng, $\mathrm{X}$. Dai, and Z. Fang, Dirac semimetal and topological phase transitions in $A_{3} \mathrm{Bi}(A=\mathrm{Na}, \mathrm{K}, \mathrm{Rb})$, Phys. Rev. B 85, 195320 (2012). 